\title{
Research Article \\ Revisited Optimal Error Bounds for Interpolatory Integration Rules
}

\author{
François Dubeau \\ Département de Mathématiques, Université de Sherbrooke, 2500 Boulevard de l'Université, \\ Sherbrooke, QC, Canada J1K 2R1 \\ Correspondence should be addressed to François Dubeau; francois.dubeau@usherbrooke.ca
}

Received 5 April 2016; Accepted 17 October 2016

Academic Editor: Slimane Adjerid

Copyright (C) 2016 François Dubeau. This is an open access article distributed under the Creative Commons Attribution License, which permits unrestricted use, distribution, and reproduction in any medium, provided the original work is properly cited.

We present a unified way to obtain optimal error bounds for general interpolatory integration rules. The method is based on the Peano form of the error term when we use Taylor's expansion. These bounds depend on the regularity of the integrand. The method of integration by parts "backwards" to obtain bounds is also discussed. The analysis includes quadrature rules with nodes outside the interval of integration. Best error bounds for composite integration rules are also obtained. Some consequences of symmetry are discussed.

\section{Introduction}

Establishing numerical integration rules and their error bounds is an old subject; for example, see the following classic textbooks [1-4]. However, in recent years, several papers have presented error bounds for the midpoint, trapezoidal, and Simpson's rules; see [5-10]. In these papers, questions were raised about the generality of the results in terms of the optimality of the bounds and regularity of the function. They also suggest that consequences of the symmetry on integration rules should be more fully investigated. Based on Peano form of the error term when we use Taylor's expansion, we present a unified way to obtain optimal error bounds for general interpolatory integration rules. These bounds depend on the regularity of the integrand. Other similar approaches based on kernels have been recently presented in [11] for Newton-Cotes quadrature rules and in [12] for Gaussian weighted quadrature rules. follows:

Let us start by a transformation of the definite integral as

$$
\int_{a}^{b} F(X) d X=\int_{-h}^{h} f(x) d x
$$

where $X=(a+b) / 2+x, h=(b-a) / 2$, and $f(x)=F((a+$ $b) / 2+x)$. Then we consider the method of undetermined coefficients to approximate the following expression:

$$
Q(f ; h)=\frac{1}{h} \int_{-h}^{h} f(x) d x .
$$

The method of undetermined coefficients consists in finding a $(n+1)$-dimensional weight vector $\vec{a}=\left(a_{0}, a_{1}, a_{2}, \ldots, a_{n}\right)$ associated with a given $(n+1)$-dimensional vector of distinct coordinates (or nodes) $\vec{x}=\left(x_{0}, x_{1}, x_{2}, \ldots, x_{n}\right)$ such that $Q(f ; h)$ is approximated by its discrete version $Q_{n}^{d}(f ; h)$ given by

$$
Q_{n}^{d}(f ; h)=\sum_{i=0}^{n} a_{i} f\left(h x_{i}\right) .
$$

The truncation error of the process is defined by

$$
R_{n}(f ; h)=Q(f ; h)-Q_{n}^{d}(f ; h),
$$

and the method is based on the requirement that

$$
R_{n}(f ; h)=o\left(h^{n}\right) .
$$


A general analysis of the method of undetermined coefficients was recently published in [13].

To study the truncation error, two approaches both based on Taylor's expansions for absolutely continuous functions and Peano's kernels are presented. In the first approach, the "direct method," we use $(4)$ for $R_{n}(f ; h)$ and a Taylor expansion of the integrand. While in the second approach, the method of integration by parts "backwards" $[4,14-16]$, we use Taylor's expansion not only for integrand but also for $W_{n}(f ; h)=h R_{n}(f ; h)$. It is shown that both methods lead to the same best error bounds.

Coming back to the definite integral given in (1), we get

$$
\int_{a}^{b} F(X) d X \approx \frac{b-a}{2} \sum_{i=0}^{n} a_{i} F\left(X_{i}\right)
$$

where $X_{i}=(a+b) / 2+((b-a) / 2) x_{i}$, for $i=0,1,2, \ldots, n$. However, to compute this definite integral we can also use a composite rule, for which we also present optimal error bounds.

The plan of the paper is the following. In Section 2, we obtain best error bounds using Taylor's expansions and Peano's kernels. Section 2.3 is devoted to the "direct method," and Section 2.4 presents the "method of integration by parts backwards." Examples are presented in Section 3. Composite integration rules is the object of Section 4. Finally we consider symmetric rules in Section 5.

We use $f^{(l)}(x)$ for the $l$ th derivative of $f(x)$ for $l=$ $0,1,2, \ldots$, where $f^{(0)}(x)=f(x)$. Let $1 \leq p \leq \infty$; if $f(x)$ is defined on a set $E,\|f\|_{p, E}$ is its $p$-norm on $E$, and if $\vec{v}$ is a vector in $\mathbb{R}^{n}$, its $p$-norm is $\|\vec{v}\|_{p}$.

\section{Truncation Error}

\subsection{Introduction. Let}

$$
\begin{aligned}
\tau & =\max \left\{1,\left|x_{0}\right|,\left|x_{1}\right|,\left|x_{2}\right|, \ldots,\left|x_{n}\right|\right\}=\max \left\{1,\|\vec{x}\|_{\infty}\right\} \\
& \geq 1
\end{aligned}
$$

and set $H=\tau h$. Let us observe that $H>h$, or $\tau>1$, means that at least one $\left|x_{i}\right|$ is strictly greater that 1 . Hence it allows the possibility of having numerical integration formulae with nodes $h x_{i}$ outside the interval of integration $[-h, h]$; see [17], for example. In that situation, the function $f(x)$ has to be defined on an interval $[-H, H]$ which contains $[-h, h]$.

For the method of undetermined coefficients it is required that $R_{n}(f ; h)=0$ at least for polynomial of degree less than or equal to $n$, but $R_{n}(f ; h)=0$ might hold for some polynomials $f(x)$ of degree higher than $n$; see [13]. It happens for Simpson's rule $(n=2)$ which is also exact for polynomials of degree 3 , or also for $n+1$ points Gaussian rule which is exact for polynomials of degree $\leq 2 n+1$. Let us define the degree of accuracy (or precision) $n_{0}$ of the approximation process (3) as the largest integer $n_{0} \geq n$ such that $R_{n}(f ; h)=0$ holds for any polynomial $f(x)$ of degree $l \leq n_{0}$. These rules are also called interpolatory quadrature formulae.
2.2. Taylor's Expansions. Let $H>0$ and $I_{H}=[-H, H]$. For $H=1$, we simply use $I=[-1,1], I_{H}^{+}=[0, H]$, and $I_{H}^{-}=[-H, 0]$. Let $p$ and $q$ be two extended real numbers such that $1 \leq p, q \leq \infty$ and $1 / p+1 / q=1$. Let $C^{l}\left(I_{H}\right)$ be the set of continuously differentiable functions up to order $l$ on $I_{H}$ and let $p \in[1,+\infty]$. Let $A C^{l+1, p}\left(I_{H}\right)$ be the set of absolutely continuous function on $I_{H}$ defined by $f \in A C^{l+1, p}\left(I_{H}\right)$ if and only if

$$
f \in C^{l}\left(I_{H}\right)
$$

and
(a) $f^{(l+1)} \in L^{p}\left(I_{H}\right)$,
(b) $f^{(l)}(s)=f^{(l)}(r)+\int_{r}^{s} f^{(l+1)}(\xi) d \xi, \forall r, s \in I_{H}$.

Taylor's expansion of $f(x) \in A C^{l+1, p}\left(I_{H}\right)$ around $x=0$ of order $l+1$ is

$$
\begin{aligned}
f(x)= & \sum_{j=0}^{l} \frac{f^{(j)}(0)}{j} x^{j} \\
& +\int_{-H}^{H} f^{(l+1)}(y) K_{T, l}(x, y ; H) d y,
\end{aligned}
$$

where $K_{T, l}(x, y ; H)$ is its associated kernel

$$
\begin{aligned}
& K_{T, l}(x, y ; H) \\
& \quad=\frac{1}{l !}\left[(x-y)_{+}^{l} \mathbf{1}_{I_{H}^{+}}(y)+(-1)^{l+1}(y-x)_{+}^{l} \mathbf{1}_{I_{H}^{-}}(y)\right],
\end{aligned}
$$

for any $x, y$ in $I_{H}$; see $[18,19]$. This kernel is a piecewise polynomial function of degree $l$. In this expression, if $E$ is a subset of $\mathbb{R}$, then

$$
\mathbf{1}_{E}(y)= \begin{cases}1 & \text { if } y \in E \\ 0 & \text { if } y \notin E,\end{cases}
$$

and for any nonnegative integer $l$

$$
(z)_{+}^{l}= \begin{cases}z^{l} & \text { if } z>0 \\ 0 & \text { if } z \leq 0 .\end{cases}
$$

If we set $H=h \tau, x=h \xi$, and $y=h \eta$, then the kernel becomes

$$
K_{T, l}(x, y ; H)=K_{T, l}(h \xi, h \eta ; h \tau)=h^{l} K_{T, l}(\xi, \eta ; \tau),
$$

for any $\xi$ and $\eta$ in $I_{\tau}$.

2.3. Direct Method. For the truncation error analysis, let $f(x) \in A C^{l+1, p}\left(I_{H}\right)$, for $0 \leq l \leq n_{0}$. Using Taylor's expansion (9) of $f(x)$ of order $l+1$ and the fact that the process is exact for polynomials of degree $\leq l$, we obtain

$$
R_{n}(f ; h)=\int_{-H}^{H} f^{(l+1)}(y) K_{n}^{l}(y ; H, h) d y .
$$


Here $K_{n}^{l}(y ; H, h)$ is the Peano kernel associated with the process $Q_{n}^{d}(f ; h)$, given by

$$
K_{n}^{l}(y ; H, h)=R_{n}\left(K_{T, l}(\cdot, y ; H) ; h\right) .
$$

Let $q \in[1,+\infty]$ be the conjugate of $p$ such that $1 / p+1 / q=1$. From Holder's inequality, we obtain

$$
\left|R_{n}(f ; h)\right| \leq\left\|f^{(l+1)}\right\|_{p, I_{H}}\left\|K_{n}^{l}(\cdot ; H, h)\right\|_{q, I_{H}},
$$

because $K_{n}^{l}(y ; H, h) \in L^{\infty}\left(I_{H}\right) \subseteq L^{q}\left(I_{H}\right)$, for any $1 \leq q \leq \infty$.

Let us observe that if $H=h \tau$ and $y=h \eta$, then $K_{n}^{l}(y ; H, h)=h^{l} K_{n}^{l}(\eta ; \tau, 1)$. It follows that

$$
\begin{aligned}
\left\|K_{n}^{l}(\cdot ; H, h)\right\|_{q, I_{H}} & =h^{l+1-1 / p}\left\|K_{n}^{l}(\cdot ; \tau, 1)\right\|_{q, I_{\tau}}, \\
\left|R_{n}(f ; h)\right| & \leq h^{l+1-1 / p} C_{n}^{l, p}\left\|f^{(l+1)}\right\|_{p, I_{H}},
\end{aligned}
$$

where the constant

$$
C_{n}^{l, p}=\left\|K_{n}^{l}(\cdot ; \tau, 1)\right\|_{q, I_{\tau}}
$$

does not depend on $h$. So we have established the following results.

Theorem 1. Let the real number $\tau \geq 1$ be fixed and $H=h \tau$. If $R_{n}(f ; h)=0$, for any polynomial of degree $\leq n_{0}$, then (16), and equivalently (18), holds for any $f \in A C^{l+1, p}\left(I_{H}\right)$, where $0 \leq l \leq n_{0}$.

Theorem 2. In (17), the kernel $K_{n}^{l}(y ; H, h)$ is given by

$$
\begin{aligned}
& K_{n}^{l}(y ; H, h)=\frac{1}{h} \int_{-h}^{h} K_{T, l}(x, y ; H) d x \\
& \quad-\sum_{i=0}^{n} a_{i} K_{T, l}\left(h x_{i}, y ; H\right)=\frac{1}{(l+1) ! h}\left[(h-y)_{+}^{l+1}\right. \\
& \left.\cdot \mathbf{1}_{I_{H}^{+}}(y)+(-1)^{l+1}(y+h)_{+}^{l+1} \mathbf{1}_{I_{H}^{-}}(y)\right]-\frac{1}{l !} \\
& \cdot \sum_{i=0}^{n} a_{i}\left[\left(h x_{i}-y\right)_{+}^{l} \mathbf{1}_{I_{H}^{+}}(y)\right. \\
& \left.+(-1)^{l+1}\left(y-h x_{i}\right)_{+}^{l} \mathbf{1}_{I_{H}^{-}}(y)\right]
\end{aligned}
$$

and, in (19), the kernel $K_{n}^{l}(\eta ; \tau, 1)$ is given by

$$
\begin{aligned}
& K_{n}^{l}(\eta ; \tau, 1)=\frac{1}{(l+1) !}\left[(1-\eta)_{+}^{l+1} \mathbf{1}_{I_{\tau}^{+}}(\eta)+(-1)^{l+1}(\eta\right. \\
& \left.\quad+1)_{+}^{l+1} \mathbf{1}_{I_{\tau}^{-}}(\eta)\right]-\frac{1}{l !} \sum_{i=0}^{n} a_{i}\left[\left(x_{i}-\eta\right)_{+}^{l} \mathbf{1}_{I_{\tau}^{+}}(\eta)\right. \\
& \left.\quad+(-1)^{l+1}\left(\eta-x_{i}\right)_{+}^{l} \mathbf{1}_{I_{\tau}^{-}}(\eta)\right]
\end{aligned}
$$

Remark 3. It can be proved that the bound given by (16) and (17), or equivalently by (18) and (19), is the best possible one; see [13].
Remark 4. For the function $F(X)$ used in (1), we have

$$
\left\|F^{(l+1)}\right\|_{p,\left[a^{\prime}, b^{\prime}\right]}=\left\|f^{(l+1)}\right\|_{p, I_{H}},
$$

where $[a, b] \subseteq\left[a^{\prime}, b^{\prime}\right]=(a+b) / 2+[-H, H]$. Obviously, $F(X)$ is supposed to be defined for any $X \in\left[a^{\prime}, b^{\prime}\right]$.

2.4. Integration by Parts "Backwards". The method of integration by parts "backwards," which is reported to have initially appeared in $[4,14-16]$, was used in [6-10] to find truncation error estimates for the midpoint, trapezoidal, and Simpson's rules. These rules possess a property of symmetry, which help in finding optimal bounds in these cases. However, the method of integration by parts "backwards" can be applied to any rule that can be obtained by the method of undetermined coefficients. We present an analysis of the truncation error based on this method.

The process is based on Taylor's expansions of

$$
W_{n}(f ; h)=h R_{n}(f ; h),
$$

and we suppose that $f \in A C^{l+1, p}\left(I_{H}\right)$ for $0 \leq l \leq n_{0}$. We have

$$
\begin{aligned}
W_{n}(f ; h) & =h R_{n}(h ; f) \\
& =\int_{-h}^{h} f(x) d x-h \sum_{i=0}^{n} a_{i} f\left(h x_{i}\right),
\end{aligned}
$$

so $W_{n}(f ; 0)=0$. For $1 \leq j<l$, we have

$$
\begin{aligned}
& W_{n}^{(j)}(f ; h) \\
& =f^{(j-1)}(h)+(-1)^{j-1} f^{(j-1)}(-h) \\
& \quad-j \sum_{i=0}^{n} a_{i} x_{i}^{j-1} f^{(j-1)}\left(h x_{i}\right)-h \sum_{i=0}^{n} a_{i} x_{i}^{j} f^{(j)}\left(h x_{i}\right), \\
& \lim _{h \rightarrow 0} W_{n}^{(j)}(f ; h) \\
& \quad=f^{(j-1)}(0)\left[1+(-1)^{j-1}-j \sum_{i=0}^{n} a_{i} x_{i}^{j-1}\right]=0 .
\end{aligned}
$$

Also, for $j=l$,

$$
\begin{aligned}
W_{n}^{(l)}(f ; h)= & f^{(l-1)}(h)+(-1)^{l-1} f^{(l-1)}(-h) \\
& -l \sum_{i=0}^{n} a_{i} x_{i}^{l-1} f^{(l-1)}\left(h x_{i}\right) \\
& -h \sum_{i=0}^{n} a_{i} x_{i}^{l} f^{(l)}\left(h x_{i}\right) .
\end{aligned}
$$

Using Taylor's expansions of order 2 for $f^{(l-1)}(x)$ which is in $A C^{2, p}\left(I_{H}\right)$ and of order 1 for $f^{(l)}(x)$ which is in $A C^{1, p}\left(I_{H}\right)$, we obtain

$$
W_{n}^{(l)}(f ; h)=\int_{-H}^{H} f^{(l+1)}(y) K_{W, l}(y ; H, h) d y,
$$


where

$$
\begin{aligned}
K_{W, l}(y ; H, h)= & K_{T, 1}(h, y ; H) \\
& +(-1)^{l-1} K_{T, 1}(-h, y ; H) \\
& -l \sum_{i=0}^{n} a_{i} x_{i}^{l-1} K_{T, 1}\left(h x_{i}, y ; H\right) \\
& -h \sum_{i=0}^{n} a_{i} x_{i}^{l} K_{T, 0}\left(h x_{i}, y ; H\right) .
\end{aligned}
$$

We remark that

$$
K_{W, l}(y ; H, h)=K_{W, l}(h \eta ; \tau h, h)=h K_{W, l}(\eta ; \tau, 1) .
$$

Taylor's expansion of order $l$ for $W_{n}(f ; h)$ or integration by parts "backwards" leads to

$$
\begin{aligned}
W_{n}(f ; h)=\int_{0}^{h} W_{n}^{(l)}(f ; z) \frac{(h-z)^{l-1}}{(l-1) !} d z \\
=\int_{0}^{h}\left[\int_{-\tau z}^{\tau z} f^{(l+1)}(y) K_{W, l}(y ; \tau z, z) d y\right] \\
\cdot \frac{(h-z)^{l-1}}{(l-1) !} d z=\int_{-\tau h}^{\tau h} f^{(l+1)}(y) \\
\cdot\left[\int_{|y| / \tau}^{h} K_{W, l}(y ; \tau z, z) \frac{(h-z)^{l-1}}{(l-1) !} d z\right] d y \\
=\int_{-H}^{H} f^{(l+1)}(y) \widehat{K}_{n}^{l}(y ; H, h) d y .
\end{aligned}
$$

As mentioned in Remark 3, the construction used in [13] to show the optimality of the bounds leads also to the following result.

Theorem 5. Let $h>0$ and $H=\tau$ h be given; the kernels $K_{n}^{l}(\cdot ; H, h)$ and $\widehat{K}_{n}^{l}(\cdot ; H, h)$ are such that

$$
h K_{n}^{l}(y ; H, h)=\widehat{K}_{n}^{l}(y ; H, h)
$$

almost everywhere.

As a consequence both methods lead to the same best error bounds.

\section{Examples}

In this section we present several examples. In some cases, constants $C_{n}^{l, p}=\left\|K_{n}^{l}(\cdot ; \tau, 1)\right\|_{q, I_{\tau}}$ are computed for $p=\infty$ (and $q=1$ ) and are compared to constants already existing in the references.

Example 1. Midpoint rule (or one point Gauss rule): $n=0$, $\vec{x}=(0)$, and hence $\tau=1$. Also, $\vec{a}=(2)$, and $n_{0}=1$. The quadrature formula is

$$
\int_{-1}^{1} f(x) d x \approx 2 f(0)
$$

For $l=0,1$, we have

$$
\begin{aligned}
& K_{0}^{l}(\eta ; 1,1)=\frac{1}{(l+1) !}\left[(1-\eta)^{l+1} \mathbf{1}_{I^{+}}(\eta)\right. \\
& \left.+(-1)^{l+1}(\eta+1)^{l+1} \mathbf{1}_{I^{-}}(\eta)\right],
\end{aligned}
$$

so we obtain

$$
\begin{aligned}
& \left\|K_{0}^{l}(\cdot ; 1,1)\right\|_{q, I} \\
& \quad= \begin{cases}\frac{1}{(l+1) !}\left(\frac{2}{1+(l+1) q}\right)^{1 / q} & \text { for } 1 \leq q<\infty \\
\frac{1}{(l+1) !} & \text { for } q=\infty .\end{cases}
\end{aligned}
$$

For example, $C_{0}^{0, \infty}=1$ and $C_{0}^{1, \infty}=1 / 3$, which correspond to values obtained in $[7,9]$.

Example 2. Trapezoidal rule: $n=1, \vec{x}=(-1,1)$, and $\tau=1$. Also, $\vec{a}=(1,1)$ and $n_{0}=1$. The quadrature formula is

$$
\int_{-1}^{1} f(x) d x \approx f(-1)+f(1) .
$$

For $l=0,1$, we have

$$
\begin{aligned}
K_{1}^{l}(\eta ; 1,1)= & -\frac{(1-\eta)^{l}}{(l+1) !}(\eta+l) \mathbf{1}_{I^{+}}(\eta) \\
& +(-1)^{l+1} \frac{(\eta+1)^{l}}{(l+1) !}(\eta-l) \mathbf{1}_{I^{-}}(\eta) .
\end{aligned}
$$

We obtain

$$
\begin{aligned}
& \left\|K_{1}^{l}(\cdot ; 1,1)\right\|_{q, I} \\
& = \begin{cases}\frac{2(l+1)}{(l+2) !} & \text { for } q=1 \\
\frac{1}{(l+1) !}\left[2 \int_{0}^{1}(1-\eta)^{l q}(\eta+l)^{q} d \eta\right]^{1 / q} & \text { for } 1<q<\infty \\
\frac{1}{(l+1) !} & \text { for } q=\infty .\end{cases}
\end{aligned}
$$

For example, $C_{0,1}^{0, \infty}=1$ and $C_{0,1}^{1, \infty}=2 / 3$, which correspond to values obtained in $[5,6,9]$.

Example 3. First Simpson's rule: $n=2, \vec{x}=(-1,0,1)$, and $\tau=1$. Also, $\vec{a}=(1 / 3,4 / 3,1 / 3)$ and $n_{0}=3$. The quadrature formula is

$$
\int_{-1}^{1} f(x) d x \approx \frac{1}{3} f(-1)+\frac{4}{3} f(0)+\frac{1}{3} f(1) .
$$

For $l=0,1,2,3$, we have

$$
\begin{aligned}
K_{2}^{l}(\eta ; 1,1)= & -\frac{(1-\eta)^{l}}{(l+1) !}\left(\eta+\frac{l-2}{3}\right) \mathbf{1}_{I^{+}}(\eta) \\
& +(-1)^{l+1} \frac{(\eta+1)^{l}}{(l+1) !}\left(\eta-\frac{l-2}{3}\right) \mathbf{1}_{I^{-}}(\eta)
\end{aligned}
$$


We obtain

$$
\left\|K_{0,2}^{l}(\cdot ; 1,1)\right\|_{q, I}= \begin{cases}\frac{1}{(l+1) !}\left[2 \int_{0}^{1}(1-\eta)^{l q}\left|\frac{\eta+(l-2)}{3}\right|^{q} d \eta\right]^{1 / q} & \text { for } 1 \leq q<\infty \\ \frac{1}{(l+1) !} \max _{\eta \in I^{+}}\left|(1-\eta)^{l}\left(\eta+\frac{(l-2)}{3}\right)\right| & \text { for } q=\infty\end{cases}
$$

For $p=\infty$ and $q=1$, we get $C_{0,2}^{0, \infty}=5 / 9, C_{0,2}^{1, \infty}=8 / 81$, $C_{0,2}^{2, \infty}=1 / 36$, and $C_{0,2}^{3, \infty}=1 / 90$, which correspond to constants obtained in $[5,7-10]$.

Example 4. Second Simpson's rule: $n=3, \vec{x}=(-1,-1 / 3$, $1 / 3,1)$, and $\tau=1$. Also, $\vec{a}=(1 / 4,3 / 4,3 / 4,1 / 4)$ and $n_{0}=3$. The quadrature formula is

$$
\begin{aligned}
\int_{-1}^{1} f(x) d x \approx & \frac{1}{4} f(-1)+\frac{3}{4} f\left(-\frac{1}{3}\right)+\frac{3}{4} f\left(\frac{1}{3}\right) \\
& +\frac{1}{4} f(1) .
\end{aligned}
$$

For $l=0,1,2,3$, we have

$$
\begin{aligned}
K_{3}^{l}(\eta ; 1,1) & \\
= & -\left[\frac{(1-\eta)^{l}}{(l+1) !}\left(\eta+\frac{l-3}{4}\right)+\frac{3}{4 l !}\left(\frac{1}{3}-\eta\right)_{+}^{l}\right] \\
\cdot & \mathbf{1}_{I^{+}}(\eta)+(-1)^{l+1} \\
\cdot & {\left[\frac{(\eta+1)^{l}}{(l+1) !}\left(\eta-\frac{l-3}{4}\right)-\frac{3}{4 l !}\left(\eta+\frac{1}{3}\right)_{+}^{l}\right] \mathbf{1}_{I^{-}}(\eta) . }
\end{aligned}
$$

Example 5. A 2-point Gauss rule: $n=1, \vec{x}=(-1 / \sqrt{3}, 1 / \sqrt{3})$, and $\tau=1$. Also, $\vec{a}=(1,1)$ and $n_{0}=3$. The quadrature formula is

$$
\int_{-1}^{1} f(x) d x \approx f\left(-\frac{1}{\sqrt{3}}\right)+f\left(\frac{1}{\sqrt{3}}\right) .
$$

For $l=0,1,2,3$, we have

$$
\begin{aligned}
K_{1}^{l} & (\eta ; 1,1) \\
= & \frac{1}{(l+1) !}\left[(1-\eta)^{l+1}-(l+1)\left(\frac{1}{\sqrt{3}}-\eta\right)_{+}^{l}\right] \\
& \cdot \mathbf{1}_{I^{+}}(\eta) \\
& +\frac{(-1)^{l+1}}{(l+1) !}\left[(\eta+1)^{l+1}-(l+1)\left(\eta+\frac{1}{\sqrt{3}}\right)_{+}^{l}\right] \\
& \cdot \mathbf{1}_{I^{-}}(\eta) .
\end{aligned}
$$

Example 6. A 3-point Gauss rule: $n=2, \vec{x}=(-\sqrt{3 / 5}, 0$, $\sqrt{3 / 5})$, and $\tau=1$. Also, $\vec{a}=(5 / 9,8 / 9,5 / 9)$ and $n_{0}=5$. The quadrature formula is

$$
\int_{-1}^{1} f(x) d x \approx \frac{5}{9} f\left(-\sqrt{\frac{3}{5}}\right)+\frac{8}{9} f(0)+\frac{5}{9} f\left(\sqrt{\frac{3}{5}}\right) .
$$

For $l=0,1,2,3,4,5$, we have

$$
\begin{aligned}
& K_{0,2}^{l}(\eta ; 1,1) \\
& \quad=\frac{1}{(l+1) !}\left[(1-\eta)^{l+1}-\frac{5}{9}(l+1)\left(\sqrt{\frac{3}{5}}-\eta\right)_{+}^{l}\right] \\
& \cdot \mathbf{1}_{I^{+}}(\eta) \\
& +\frac{(-1)^{l+1}}{(l+1) !}\left[(\eta+1)^{l+1}-\frac{5}{9}(l+1)\left(\eta+\sqrt{\frac{3}{5}}\right)_{+}^{l}\right] \\
& \cdot \mathbf{1}_{I^{-}}(\eta) .
\end{aligned}
$$

Example 7. First nonstandard rule: $n=1, \vec{x}=(-2,2)$, and $\tau=2$. Also, $\vec{a}=(1,1)$ and $n_{0}=1$. The quadrature formula is

$$
\int_{-1}^{1} f(x) d x \approx f(-2)+f(2) \text {. }
$$

For $l=0,1$, we have

$$
\begin{aligned}
K_{1}^{l}(\eta ; 2,1) & \\
= & \frac{1}{(l+1) !}\left[(1-\eta)_{+}^{l+1}-(l+1)(2-\eta)_{+}^{l}\right] \mathbf{1}_{I_{2}^{+}}(\eta) \\
& +\frac{(-1)^{l+1}}{(l+1) !}\left[(\eta+1)_{+}^{l+1}-(l+1)(\eta+2)_{+}^{l}\right] \mathbf{1}_{I_{2}^{-}}(\eta) .
\end{aligned}
$$

Example 8. Second nonstandard rule: $n=2, \vec{x}=(-\sqrt{2}$, $0, \sqrt{2})$, and $\tau=\sqrt{2}$. Also, $\vec{a}=(1 / 6,10 / 6,1 / 6)$ and $n_{0}=3$. The quadrature formula is

$$
\int_{-1}^{1} f(x) d x \approx \frac{1}{6} f(-\sqrt{2})+\frac{10}{6} f(0)+\frac{1}{6} f(\sqrt{2}) .
$$

For $l=0,1,2,3$, we have

$$
\begin{aligned}
& K_{2}^{l}(\eta ; \sqrt{2}, 1) \\
& \quad=\frac{1}{(l+1) !}\left[(1-\eta)_{+}^{l+1}-\frac{(l+1)}{6}(\sqrt{2}-\eta)_{+}^{l}\right] \mathbf{1}_{I_{\sqrt{2}}^{+}}(\eta) \\
& \quad+\frac{(-1)^{l+1}}{(l+1) !}\left[(\eta+1)_{+}^{l+1}-\frac{(l+1)}{6}(\eta+\sqrt{2})_{+}^{l}\right] \\
& \quad \cdot \mathbf{1}_{I_{\sqrt{2}}^{-}}(\eta) .
\end{aligned}
$$


Example 9. Third nonstandard rule: $n=2, \vec{x}=(2,0,2)$, and $\tau=2$. Also, $\vec{a}=(1 / 12,11 / 6,1 / 12)$ and $n_{0}=3$. The quadrature formula is

$$
\int_{-1}^{1} f(x) d x \approx \frac{1}{12} f(-2)+\frac{11}{6} f(0)+\frac{1}{12} f(2) .
$$

For $l=0,1,2,3$, we have

$$
\begin{aligned}
& K_{2}^{l}(\eta ; 2,1) \\
& =\frac{1}{(l+1) !}\left[(1-\eta)_{+}^{l+1}-\frac{(l+1)}{12}(2-\eta)_{+}^{l}\right] \mathbf{1}_{I_{2}^{+}}(\eta) \\
& \quad+\frac{(-1)^{l+1}}{(l+1) !}\left[(\eta+1)_{+}^{l+1}-\frac{(l+1)}{12}(\eta+2)_{+}^{l}\right] \mathbf{1}_{I_{2}^{-}}(\eta) .
\end{aligned}
$$

Example 10. Gauss-Radau rule: $n=1, \vec{x}=(-1 / 3,1)$, and $\tau=$ 1. Also, $\vec{a}=(3 / 2,1 / 2)$ and $n_{0}=2$. The quadrature formula is

$$
\int_{-1}^{1} f(x) d x \approx \frac{3}{2} f\left(-\frac{1}{3}\right)+\frac{1}{2} f(1) \text {. }
$$

For $l=0,1,2$, we have

$$
\begin{aligned}
& K_{1}^{l}(\eta ; 1,1)=-\frac{(1-\eta)^{l}}{(l+1) !}\left(\eta+\frac{l-1}{2}\right) \mathbf{1}_{I^{+}}(\eta) \\
& +\frac{(-1)^{l+1}}{(l+1) !}\left[(\eta+1)^{l+1}-\frac{3}{2}(l+1)\left(\eta+\frac{1}{3}\right)_{+}^{l}\right] \\
& \cdot \mathbf{1}_{I^{-}}(\eta) .
\end{aligned}
$$

\section{Composite Rules}

For an integral $\int_{a}^{b} f(X) d X$, where $f \in A C^{l+1, p}([a, b])$, a composite rule uses a partition of $[a, b]$ in $M$ subintervals and applies a formula on each subinterval. To simplify, we consider subintervals of equal length $h=(b-a) / 2 M$. To allow the possibility that $\tau>1$, which cause an overlap between subintervals, we suppose also that $f \in A C^{l+1, p}\left(\left[a^{\prime}, b^{\prime}\right]\right)$, where $a^{\prime}<a<b<b^{\prime}$ and $[a-(\tau-1) h, b+(\tau-1) h] \in\left[a^{\prime}, b^{\prime}\right]$, for $h$ small enough or equivalently $M$ large enough. Let us set $\xi_{m}=a+2 m h$ for $m=0,1,2, \ldots, M$, and $c_{m}=\left(\xi_{m-1}+\xi_{m}\right) / 2$, for $m=1,2,3, \ldots, M$. Then

$$
\begin{aligned}
\int_{a}^{b} f(X) d X & =\sum_{m=1}^{M} \int_{\xi_{m-1}}^{\xi_{m}} f(\xi) d \xi \\
& =\sum_{m=1}^{M} \int_{-h}^{h} f\left(c_{m}+x\right) d x \\
& =h \sum_{m=1}^{M} Q\left(f\left(c_{m}+\cdot\right) ; h\right) .
\end{aligned}
$$

The composite rule $C Q_{n}(f)$ is then defined by

$$
C Q_{n}(f)=h \sum_{m=1}^{M} Q_{n}^{d}\left(f\left(c_{m}+\cdot\right) ; h\right) .
$$

The truncation error is

$$
\begin{aligned}
& \int_{a}^{b} f(X) d X-h \sum_{m=1}^{M} Q_{n}^{d}\left(f\left(c_{m}+\cdot\right) ; h\right) \\
& \quad=h \sum_{m=1}^{M} R_{n}\left(f\left(c_{m}+\cdot\right) ; h\right) .
\end{aligned}
$$

But

$$
\begin{aligned}
& \left|\sum_{m=1}^{M} R_{n}\left(f\left(c_{m}+\cdot\right) ; h\right)\right| \leq \sum_{m=1}^{M}\left|R_{n}\left(f\left(c_{m}+\cdot\right) ; h\right)\right| \\
& \quad \leq \sum_{m=1}^{M} h^{l+1-1 / p} C_{n}^{l, p}\left\|f^{(l+1)}\right\|_{p, c_{m}+I_{H}} \\
& \quad \leq h^{l+1-1 / p} C_{n}^{l, p} \sum_{m=1}^{M}\left\|f^{(l+1)}\right\|_{p, c_{m}+I_{H}} .
\end{aligned}
$$

To measure the overlap for $1 \leq p<\infty$, let us define $N_{p}(\tau)$ by

$$
N_{p}(\tau)= \begin{cases}\lceil\tau\rceil & \text { for } 1 \leq p<\infty \\ 1 & \text { for } p=\infty\end{cases}
$$

then

$$
\begin{aligned}
& \sum_{m=1}^{M}\left\|f^{(l+1)}\right\|_{p, c_{m}+I_{H}} \\
& \quad \leq \begin{cases}M^{1-1 / p}\left[\sum_{m=1}^{M}\left\|f^{(l+1)}\right\|_{p, c_{m}+I_{H}}^{p}\right]^{1 / p} & (1 \leq p<\infty) \\
M\left\|f^{(l+1)}\right\|_{\infty\left[a^{\prime}, b^{\prime}\right],} & (p=\infty)\end{cases} \\
& \quad \leq M^{1-1 / p} N_{p}(\tau)\left\|f^{(l+1)}\right\|_{p,\left[a^{\prime}, b^{\prime}\right]},
\end{aligned}
$$

and we obtain

$$
\begin{aligned}
& \left|\sum_{m=1}^{M} R_{n}\left(f\left(c_{m}+\cdot\right) ; h\right)\right| \\
& \quad \leq h^{l}\left(\frac{b-a}{2}\right)^{1-1 / p} C_{n}^{l, p} N_{p}(\tau)\left\|f^{(l+1)}\right\|_{p,\left[a^{\prime}, b^{\prime}\right]} .
\end{aligned}
$$

\section{Symmetric Interpolatory Rules}

In case of symmetry with respect to $x=0$, more precisely when

$$
x_{i}=-x_{n-i}, \quad \text { for } i=0,1,2, \ldots, n \text {, }
$$

we have

$$
a_{i}=a_{n-i}, \quad \text { for } i=0,1,2, \ldots, n \text {. }
$$

As a consequence, we have $K_{n}^{l}(-\eta ; \tau, 1)=(-1)^{l+1} K_{n}^{l}(\eta ; \tau, 1)$, for $l=0, \ldots, n_{0}$. Moreover, $R_{n}(f ; h)=0$ for any monomials of odd degree; then $n_{0}$ is odd and $K_{0, n}^{n_{0}}(\eta ; \tau, 1)$ is an even function. Moreover, for $f(x)=x^{j}$, since $f^{(l+1)}(x)=$ $(j)_{l+1} x^{j-(l+1)}$, for two nonnegative integers $j$ and $l$, it follows that 


$$
R_{n}\left(x^{j} ; h\right)= \begin{cases}0 & \text { for } j \leq n_{0}, \\ 0 & \text { for } j>n_{0} \text { and } j \text { odd } \\ 2 h^{j}(j)_{n_{0}+1} \int_{0}^{1} \eta^{j-\left(n_{0}+1\right)} K_{0, n}^{n_{0}}(\eta ; \tau, 1) d \eta & \text { for } j>n_{0} \text { and } j \text { even. }\end{cases}
$$

For some polynomials, we can evaluate exactly the truncation error, as mentioned in [10] for Simpson's rule. Indeed, let

$$
f(x)=p_{n_{0}+2}(x)=p_{n_{0}}(x)+\alpha_{n_{0}+1} x^{n_{0}+1}+\alpha_{n_{0}+2} x^{n_{0}+2},
$$

where $p_{n_{0}}(x)$ is a polynomial of degree $\leq n_{0}$. Then we have

$$
p_{n_{0}+2}^{\left(n_{0}+1\right)}(x)=\left(n_{0}+1\right) ! \alpha_{n_{0}+1}+\left(n_{0}+2\right) ! \alpha_{n_{0}+2} x .
$$

It follows, for the composite rule, that

$$
\begin{aligned}
& R_{n}\left(\left(c_{m}+\cdot\right)^{n_{0}+1} ; h\right)=\left(n_{0}+1\right) ! \int_{-H}^{H} K_{0, n}^{n_{0}}(y ; H, h) d y \\
& \quad=\left(n_{0}+1\right) ! h^{n_{0}+1} \int_{-\tau}^{\tau} K_{0, n}^{n_{0}}(\eta ; \tau, 1) d \eta \\
& \quad=2\left(n_{0}+1\right) ! h^{n_{0}+1} \int_{0}^{\tau} K_{0, n}^{n_{0}}(\eta ; \tau, 1) d \eta,
\end{aligned}
$$

$$
\begin{aligned}
& R_{n}\left(\left(c_{m}+\cdot\right)^{n_{0}+2} ; h\right)=\left(n_{0}\right. \\
& \quad+2) ! \int_{-\tau}^{\tau}\left(c_{m}+y\right) K_{n}^{n_{0}}(y ; H, h) d y \\
& \quad=\left(n_{0}\right. \\
& \quad+2) !\left[c_{m} \int_{-\tau}^{\tau} K_{n}^{n_{0}}(y ; H, h) d y+\int_{-\tau}^{\tau} y K_{n}^{n_{0}}(y ; H, h) d y\right] \\
& \quad=2\left(n_{0}+2\right) ! c_{m} h^{n_{0}+1} \int_{0}^{\tau} K_{n}^{n_{0}}(\eta ; \tau, 1) d \eta .
\end{aligned}
$$

So we have

$$
\begin{aligned}
& R_{n}\left(p_{n_{0}+2}\left(c_{m}+\cdot\right) ; h\right)=\alpha_{n_{0}+1} R_{n}\left(\left(c_{m}+\cdot\right)^{n_{0}+1} ; h\right) \\
& \quad+\alpha_{n_{0}+2} R_{n}\left(\left(c_{m}+\cdot\right)^{n_{0}+2} ; h\right)=2\left(n_{0}\right. \\
& \quad+1) ! h^{n_{0}+1}\left[\alpha_{n_{0}+1}+\left(n_{0}+2\right) \alpha_{n_{0}+2} c_{m}\right] \int_{0}^{\tau} K_{n}^{n_{0}}(\eta ; \tau, 1) d \eta .
\end{aligned}
$$

Moreover we obtain

$$
\begin{aligned}
h \sum_{m=1}^{M} R_{0, n}\left(p_{n_{0}+2}\left(c_{m}+\cdot\right) ; h\right) & =2\left(n_{0}+1\right) ! h^{n_{0}+2}\left[\alpha_{n_{0}+1} M+\left(n_{0}+2\right) \alpha_{n_{0}+2} \sum_{m=1}^{M} c_{m}\right] \int_{0}^{\tau} K_{n}^{n_{0}}(\eta ; \tau, 1) d \eta \\
& =2\left(n_{0}+1\right) ! h^{n_{0}+1}\left(\frac{b-a}{2}\right)\left[\alpha_{n_{0}+1}+\left(n_{0}+2\right) \alpha_{n_{0}+2}\left(\frac{a+b}{2}\right)\right] \int_{0}^{\tau} K_{n}^{n_{0}}(\eta ; \tau, 1) d \eta
\end{aligned}
$$

because

$$
\sum_{m=1}^{M} c_{m}=\frac{a+b}{2} M .
$$

Then we have a general explanation of the result mentioned in [10] for Simpson's rule applied to quartic and quintic polynomials.

Example 11. Midpoint rule: $n=0, \tau=1$, and $n_{0}=1$;

$$
\int_{0}^{1} K_{0}^{1}(\eta ; 1,1) d \eta=\frac{1}{6}
$$

and for $p_{3}(x)$ we have

$$
\begin{aligned}
& \int_{a}^{b} p_{3}(\xi) d \xi-C Q_{0}\left(p_{3}\right) \\
& \quad=\frac{1}{12}\left[\alpha_{2}+3 \alpha_{3}\left(\frac{a+b}{2}\right)\right] \frac{(b-a)^{3}}{M^{2}} .
\end{aligned}
$$

Example 12. Trapezoidal rule: $n=1, \tau=1$, and $n_{0}=1$;

$$
\int_{0}^{1} K_{1}^{1}(\eta ; 1,1) d \eta=-\frac{1}{3}
$$

and for $p_{3}(x)$ we have

$$
\begin{aligned}
& \int_{a}^{b} p_{3}(\xi) d \xi-C Q_{1}\left(p_{3}\right) \\
& \quad=-\frac{1}{6}\left[\alpha_{2}+3 \alpha_{3}\left(\frac{a+b}{2}\right)\right] \frac{(b-a)^{3}}{M^{2}} .
\end{aligned}
$$

Example 13 (see [10]). First Simpson $(1 / 3,4 / 3,1 / 3)$ rule: $n=$ $2, \tau=1$, and $n_{0}=3$;

$$
\int_{0}^{1} K_{2}^{3}(\eta ; 1,1) d \eta=-\frac{1}{180}
$$


and for $p_{5}(x)$ we have

$$
\begin{aligned}
& \int_{a}^{b} p_{5}(\xi) d \xi-C Q_{2}\left(p_{5}\right) \\
& \quad=-\frac{1}{120}\left[\alpha_{4}+5 \alpha_{5}\left(\frac{a+b}{2}\right)\right] \frac{(b-a)^{5}}{M^{4}} .
\end{aligned}
$$

Example 14. Second Simpson $(1 / 4,3 / 4,3 / 4,1 / 4)$ rule: $n=3$, $\tau=1$, and $n_{0}=3$;

$$
\int_{0}^{1} K_{0,3}^{3}(\eta ; 1,1) d \eta=-\frac{1}{405}
$$

and for $p_{5}(x)$ we have

$$
\begin{aligned}
& \int_{a}^{b} p_{5}(\xi) d \xi-C Q_{3}\left(p_{5}\right) \\
& \quad=-\frac{1}{270}\left[\alpha_{4}+5 \alpha_{5}\left(\frac{a+b}{2}\right)\right] \frac{(b-a)^{5}}{M^{4}} .
\end{aligned}
$$

Example 15. First nonstandard rule: $n=1, \tau=2$, and $n_{0}=1$;

$$
\int_{0}^{2} K_{1}^{1}(\eta ; 2,1) d \eta=-\frac{17}{6}
$$

and for $p_{3}(x)$ we have

$$
\begin{aligned}
& \int_{a}^{b} p_{3}(\xi) d \xi-C Q_{1}\left(p_{3}\right) \\
& \quad=-\frac{17}{2}\left[\alpha_{2}+3 \alpha_{3}\left(\frac{a+b}{2}\right)\right] \frac{(b-a)^{3}}{M^{2}} .
\end{aligned}
$$

Similar results for monomial of degree $k \geq n_{0}+2$ require the evaluation of $\sum_{m=1}^{M} c_{m}^{l}$ for $l \geq 2$, which is not simple.

\section{Competing Interests}

The author declares that he has no competing interests.

\section{Acknowledgments}

This work has been financially supported by an individual discovery grant from NSERC (Natural Sciences and Engineering Research Council of Canada).

\section{References}

[1] G. Dahlquist and Å. Bjïork, Numerical Methods in Scientific Computing, vol. 1, SIAM, Philadelphia, Pa, USA, 2008.

[2] P. J. Davis and P. Rabinowitz, Method of Numerical Integration, Academic Press, Miami, Fla, USA, 2nd edition, 1984.

[3] H. Engels, Numerical Quadrature and Cubature, Academic Press, London, UK, 1980.

[4] A. Ghizzetti and A. Ossicini, Quadrature Formulae, Academic Press, New York, NY, USA, 1970.

[5] D. Cruz-Uribe and C. J. Neugebauer, "Sharp error bounds for the trapezoidal rule and Simpson's rule," Journal of Inequalities in Pure and Applied Mathematics, vol. 3, no. 4, article 49, 2002.
[6] D. Cruz-Uribe and C. J. Neugebauer, "An elementary proof of error estimates for the trapezoidal rule," Mathematics Magazine, vol. 76, no. 4, pp. 303-306, 2003.

[7] E. C. Fazekas Jr. and P. R. Mercer, "Elementary proofs of error estimates for the midpoint and Simpson's rules," Mathematics Magazine, vol. 82, no. 5, pp. 365-370, 2009.

[8] D. D. Hai and R. C. Smith, "An elementary proof of the error estimates in Simpson's rule," Mathematics Magazine, vol. 81, no. 4, pp. 295-300, 2008.

[9] F. Sandomierski, "Unified proofs of the error estimates for the midpoint, trapezoidal, and Simpson's rules," Mathematics Magazine, vol. 86, no. 4, pp. 261-264, 2013.

[10] L. A. Talman, "Simpson's rule is exact for quintics," The American Mathematical Monthly, vol. 113, no. 2, pp. 144-155, 2006.

[11] M. Masjed-Jamei, "Unified error bounds for all Newton-Cotes quadrature rules," Journal of Numerical Mathematics, vol. 23, no. 1, pp. 67-80, 2015.

[12] M. Masjed-Jamei and I. Area, "Error bounds for Gaussian quadrature rules using linear kernels," International Journal of Computer Mathematics, vol. 93, no. 9, pp. 1505-1523, 2016.

[13] F. Dubeau, "The method of undetermined coefficients: general approach and optimal error bounds," Journal of Mathematical Analysis, vol. 5, no. 4, pp. 1-11, 2014.

[14] G. Peano, "Resto nelle formule di quadratura espresso con un integrale definito," Atti della Accademia d'Italia, Rendiconti della Classe di Scienze Fisiche Matematiche e Naturali, vol. 5, no. 22, pp. 562-569, 1913.

[15] R. von Mises, “Über allgemeine quadraturformeln," Journal für die Reine und Angewandte Mathematik, vol. 174, pp. 56-67, 1935, Reprinted in Selected Papers of Richard von Mises, vol. 1, pp. 559-574, American Mathematical Society, Providence, RI, USA, 1963.

[16] M. Bourdeau and J. Gélinas, Analyse Numérique Élémentaire, Edited by G. Morin, Montréal, Canada, 2nd edition, 1987.

[17] J. Garloff, W. Solak, and Z. Szydelko, "New integration formulas which use nodes outside the integration interval," Journal of the Franklin Institute, vol. 321, no. 3, pp. 115-126, 1986.

[18] E. Asplund and L. Bungart, A First Course in Integration, Holt, Rinehart and Winston, New York, NY, USA, 1966.

[19] L. L. Schumaker, Spline Functions: Basic Theory, John Wiley \& Sons, New York, NY, USA, 1981. 


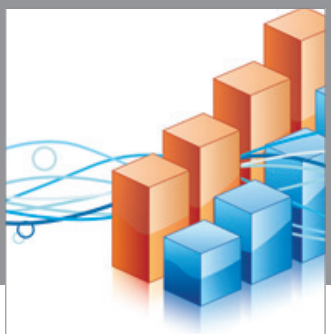

Advances in

Operations Research

vatem alat4

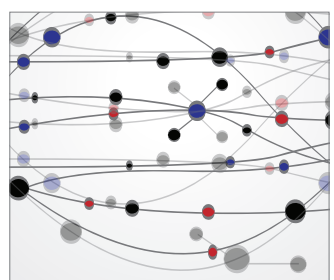

\section{The Scientific} World Journal
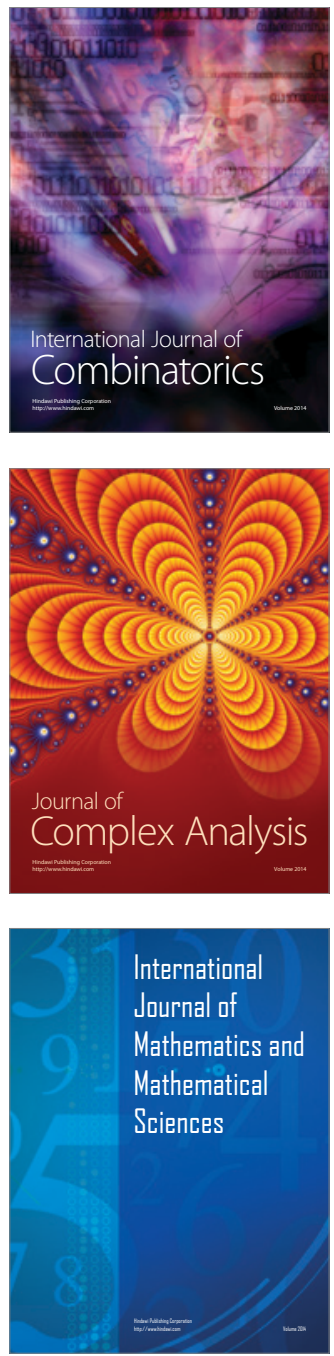
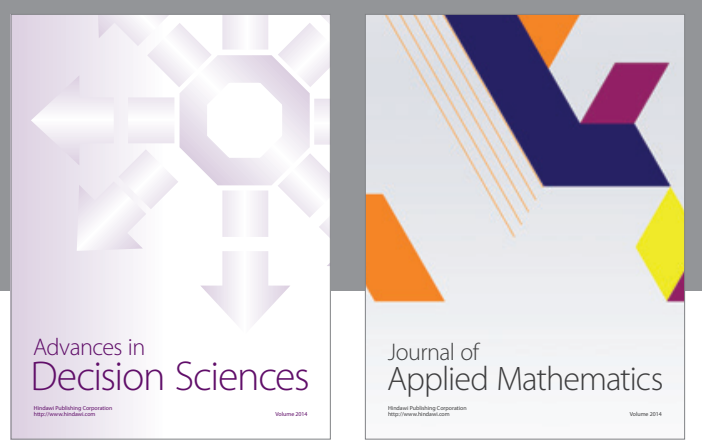

Algebra

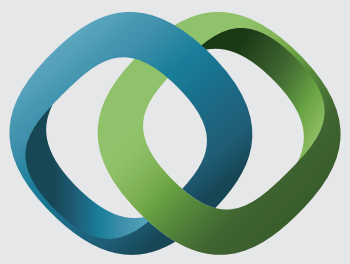

\section{Hindawi}

Submit your manuscripts at

http://www.hindawi.com
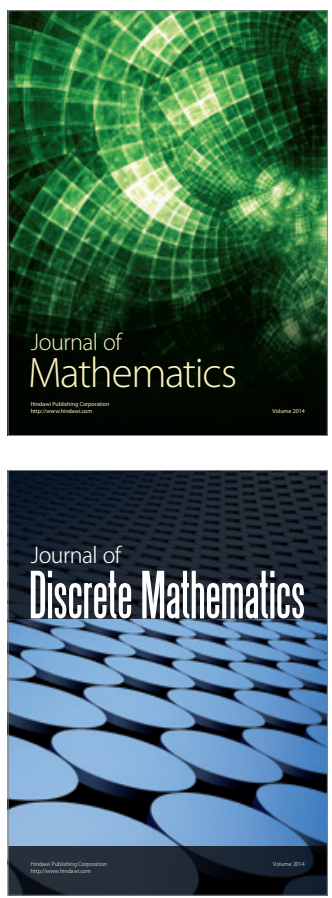

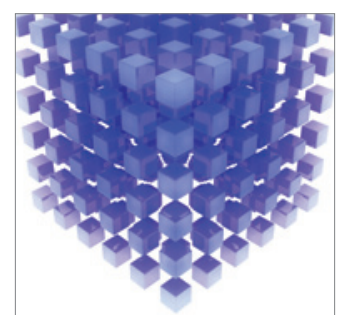

Mathematical Problems in Engineering
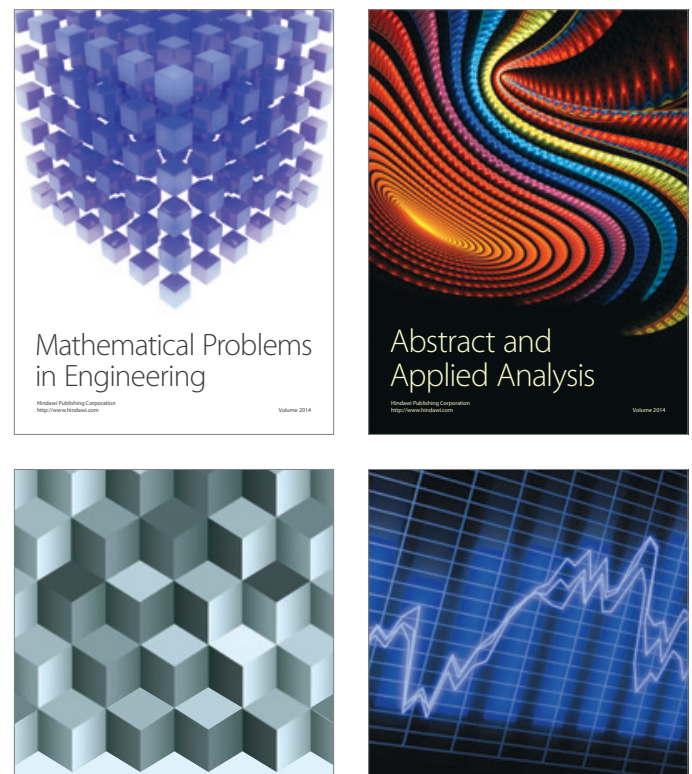

Journal of

Function Spaces

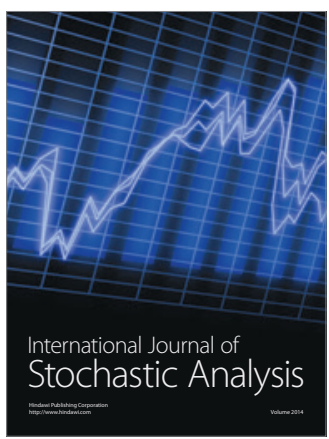

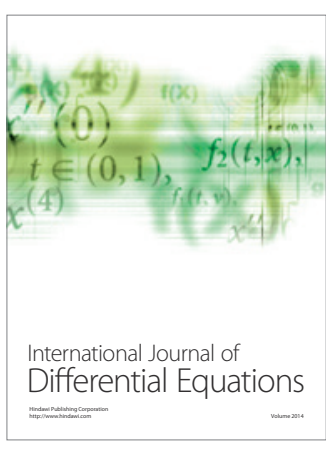
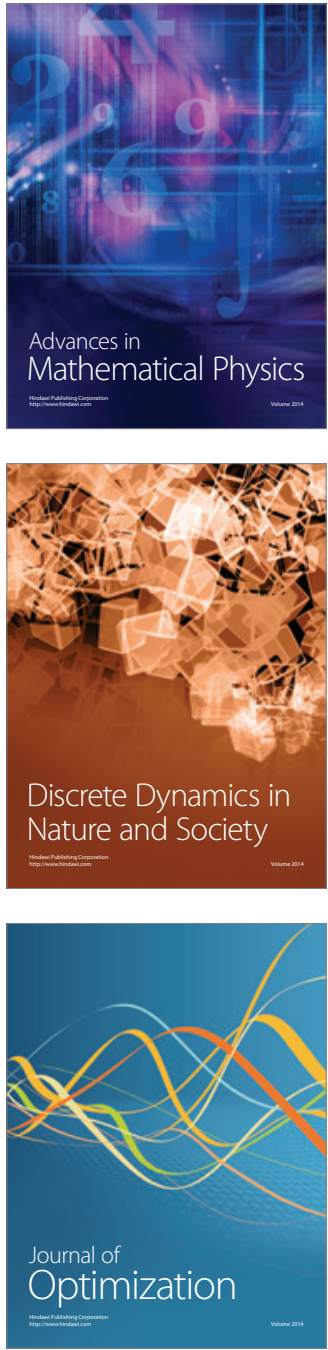